\title{
ASSESSMENT OF PROGNOSIS IN PATIENTS WITH INVASIVE BREAST CANCER AND METASTATIC SENTINEL LYMPH NODE TREATED WITH OR WITHOUT AXILLARY LYMPHADENECTOMY
}

Vanessa Monteiro Sanvido1, Simone Elias¹, Gil Facina1, Afonso Celso Pinto Nazário²

${ }^{1}$ Escola Paulista de Medicina da Universidade Federal de São Paulo - São Paulo (SP), Brazil. Universidade Federal de São Paulo - São Paulo (SP), Brazil.

Introduction: The American College of Surgeons Oncology Group (ACOSOG) Z0011 trial was a landmark in axillary surgical treatment. Its results brought significant contributions to the reduction of the extension of breast cancer surgery. However, this approach was questioned at first. Objectives: To assess the global survival and the locoregional recurrence in patients with metastatic sentinel lymph node biopsy treated with or without axillary lymphadenectomy (AL). Method: A historical cohort study was carried out with patients with primary invasive breast carcinoma and clinically negative axilla who underwent breast-conserving surgery and SLNB between February 2008 and December 2018. Results: We included 415 patients who were submitted to conserving surgery. In 23.3\% (97 patients), SLNB was positive, 56 patients were submitted only to SLNB, and 41 patients were treated with AL. The groups were homogeneous regarding the variables: age group ( $\mathrm{p}=0.279)$, anatomopathological diagnosis $(\mathrm{p}=0.210)$, histological grade $(\mathrm{p}=0.983)$, hormone receptor expression ( $\mathrm{p}=0.708)$, HER 2 expression ( $\mathrm{p}=0.695$ ) and $\mathrm{pT}(\mathrm{p}=0.334)$. Global survival, in the mean period of 5 years, was $80.1 \%$ in the SLNB group, and $87.5 \%$ in the AL group ( $\mathrm{p}=0.376$ ). The locoregional recurrence was a rare event; in the mean period of 5 years, there was $1.8 \%$ in the SLNB group, and $7.7 \%$ in the AL group ( $p=0.196)$. Only 4 locoregional recurrences were described, and all took place in up to 18 months of follow-up. Conclusions: Global survival and locoregional recurrence in patients with metastatic axillary sentinel lymph node treated only with sentinel lymph node biopsy did not present differences in relation to patients who underwent axillary lymphadenectomy, which corroborates the data from ACOSOG Z0011. The omission of axillary lymphadenectomy and the implantation of such a practice in our service benefitted the patients treated with less aggressive surgery, and, potentially, with lower morbidity. 